Erratum to:

\title{
Asymptotic Tail Probability of Randomly Weighted Sums of Dependent Random Variables with Dominated Variation
}

\section{Hai-zhong Yang}

School of Statistics, Xi'an University of Finance and Economics, Xi'an 710100, China (E-mail: h.yang@163.com)

Erratum to: Acta Mathematicae Applicatae Sinica, English Series

DOI: $10.1007 / \mathrm{s} 10255-009-8155-9$

The original version of this article unfortunately contained a mistake. Equation (3.5) should be corrected as follows:

$$
\begin{aligned}
P\left(\left|\theta_{i} X_{i}\right|>x_{i}, \theta_{j} X_{j}>x_{j}\right)= & \left(\iint_{s>t}+\iint_{s \leq t}\right) P\left(\left|\theta_{i} X_{i}\right|>\frac{x_{i}}{s}, \theta_{j} X_{j}>\frac{x_{j}}{t}\right) d G_{i j}(s, t) \\
\leq & \iint_{s>t} P\left(\left|X_{i}\right|>\frac{x_{i}}{s}, X_{j}>\frac{x_{j}}{s}\right) d G_{i j}(s, t) \\
& +\iint_{s \leq t} P\left(\left|X_{i}\right|>\frac{x_{i}}{t}, X_{j}>\frac{x_{j}}{t}\right) d G_{i j}(s, t) \\
\leq & \int_{0}^{\infty} P\left(\left|X_{i}\right|>\frac{x_{i}}{s}, X_{j}>\frac{x_{j}}{s}\right) d G_{i}(s) \\
& +\int_{0}^{\infty} P\left(\left|X_{i}\right|>\frac{x_{i}}{t}, X_{j}>\frac{x_{j}}{t}\right) d G_{j}(t) \\
= & I_{1}+I_{2} .
\end{aligned}
$$

